\title{
Hamiltonian paths and cycles in hypertournaments *
}

\author{
Gregory Gutin \\ Department of Maths and Stats \\ Brunel University, Uxbridge, Middx \\ UB8 3PH, U.K., z.g.gutin@brunel.ac.uk \\ Anders $\mathrm{Yeo}^{\dagger}$ \\ Department of Mathematics and Computer Science \\ Odense University, Denmark
}

\begin{abstract}
Given two integers $n$ and $k, n \geq k>1$, a $k$-hypertournament $T$ on $n$ vertices is a pair $(V, A)$, where $V$ is a set of vertices, $|V|=n$ and $A$ is a set of $k$-tuples of vertices, called arcs, so that for any $k$-subset $S$ of $V, A$ contains exactly one of the $k ! k$-tuples whose entries belong to $S$. A 2-hypertournament is merely an (ordinary) tournament. A path is a sequence $v_{1} a_{1} v_{2} a_{2} v_{3} \ldots v_{t-1} a_{t-1} v_{t}$ of distinct vertices $v_{1}, v_{2}, \ldots, v_{t}$ and distinct arcs $a_{1}, \ldots, a_{t-1}$ such that $v_{i}$ precedes $v_{i+1}$ in $a_{i}, 1 \leq i \leq t-1$. A cycle can be defined analogously. A path or cycle containing all vertices of $T$ (as $v_{i}$ 's) is Hamiltonian. $T$ is strong if $T$ has a path from $x$ to $y$ for every choice of distinct $x, y \in V$. We prove that every $k$-hypertournament on $n(>k)$ vertices has a Hamiltonian path (an extension of Redei's theorem on tournaments) and every strong $k$-hypertournament with $n(>k+1)$ vertices has a Hamiltonian cycle (an extension of Camion's theorem on tournaments). Despite the last result, it is shown that the Hamiltonian cycle problem remains polynomial time solvable only for $k \leq 3$ and becomes NP-complete for every fixed integer $k \geq 4$.
\end{abstract}

\section{Introduction, terminology and notation}

Hypertournaments have been studied by a number of authors (cf. Assous [1], Barbut and Bialostocki [2, 3], Bialostocki [5], Frankl [6] and Marshall [9, 10]). Reid [12] (Section 8) describes several results on hypertournaments obtained by the authors above and poses some interesting problems on the topic. In particular, he raises the problem of extending the most important results on tournaments to hypertournaments.

\footnotetext{
* This paper is dedicated to the memory of Paul Erdős.
}

†The e-mail address of AY is gyeo@imada.ou.dk 
In this paper, we obtain extensions of two of the most basic theorems on tournaments: every tournament has a Hamiltonian path (Redei's theorem), and every strong tournament has a Hamiltonian cycle (Camion's theorem) [11]. We prove that every $k$-hypertournament on $n(>k)$ vertices has a Hamiltonian path and every strong $k$-hypertournament on $n \geq k+2 \geq 5$ vertices contains a Hamiltonian cycle. We also describe an infinite family of strong $k$-hypertournaments on $k+1 \geq 4$ vertices which have no Hamiltonian cycles. We consider the complexity of the Hamiltonian cycle problem for $k$-hypertournaments and prove that the problem remains polynomial time solvable when $k=3$ and becomes NP-complete for every fixed integer $k \geq 4$.

Given two integers $n$ and $k, n \geq k>1$, a $k$-hypertournament $T$ on $n$ vertices is a pair $(V, A)$, where $V$ is a set of vertices, $|V|=n$ and $A$ is a set of $k$-tuples of vertices, called arcs, so that for any $k$-subset $S$ of $V, A$ contains exactly one of the $k$ ! $k$-tuples whose entries belong to $S$. That is, $T$ may be thought of as arising from an orientation of the hyperedges of the complete $k$-uniform hypergraph. Clearly, a 2-hypertournament is merely a tournament. For an $\operatorname{arc} a$ of $T, \bar{a}$ denotes the set of vertices contained in $a$.

Let $T=(V, A)$ denote a $k$-hypertournament $T$ on $n$ vertices. A path in $T$ is a sequence $v_{1} a_{1} v_{2} a_{2} v_{3} \ldots v_{t-1} a_{t-1} v_{t}$ of distinct vertices $v_{1}, v_{2}, \ldots, v_{t}, t \geq 1$, and distinct arcs $a_{1}, \ldots, a_{t-1}$ such that $v_{i}$ precedes $v_{i+1}$ in $a_{i}, 1 \leq i \leq t-1$. A cycle in $T$ is a sequence $v_{1} a_{1} v_{2} a_{2} v_{3} \ldots v_{t-1} a_{t-1} v_{t} a_{t} v_{1}$ of distinct vertices $v_{1}, v_{2}, \ldots, v_{t}$ and distinct $\operatorname{arcs} a_{1}, \ldots, a_{t}, t \geq 1$, such that $v_{i}$ precedes $v_{i+1}$ in $a_{i}, 1 \leq i \leq t\left(a_{t+1}=a_{1}\right)$. The above definitions of a path and cycle in a hypertournament are oriented analogs of the corresponding definitions of a path and cycle in a hypergraph (cf. $[4,10])$.

For a path or cycle $Q, V(Q)$ and $A(Q)$ denote the set of vertices ( $v_{i}$ 's above) and the set of $\operatorname{arcs}\left(a_{j}\right.$ 's above), respectively. For a pair of vertices $v_{i}$ and $v_{j}$ of a path or cycle $Q$, $Q\left[v_{i}, v_{j}\right]$ denotes the subpath of $Q$ from $v_{i}$ to $v_{j}$ (which can be empty). A path or cycle $Q$ in $T$ is Hamiltonian if $V(Q)=V(T)$. $T$ is Hamiltonian if it has a Hamiltonian cycle. A path from $x$ to $y$ is an $(x, y)$-path. $T$ is called strong if $T$ has an $(x, y)$-path for every (ordered) pair $x, y$ of distinct vertices in $T$.

We also consider paths and cycles in digraphs which will be denoted as sequences of the corresponding vertices.

For a pair of distinct vertices $x$ and $y$ in $T, A_{T}(x, y)$ denotes the set of all $\operatorname{arcs}$ of $T$ in which $x$ precedes $y$. Clearly, for all distinct $x, y \in V(T)$,

$$
\left|A_{T}(x, y)\right|+\left|A_{T}(y, x)\right|=\left(\begin{array}{l}
n-2 \\
k-2
\end{array}\right) .
$$




\section{Hamiltonian paths}

Clearly, no $k$-hypertournament with $k \geq 3$ vertices has a Hamiltonian path. However, all other hypertournaments have Hamiltonian paths:

Theorem 2.1 Every $k$-hypertournament with $n(>k)$ vertices contains a Hamiltonian path.

Proof: Let $T=(V, A)$ be a $k$-hypertournament $T$ on $n$ vertices $1,2, \ldots, n$. We consider the cases $k=n-1$ and $k<n-1$ separately.

Case 1: $k=n-1$. We proceed by induction on $k \geq 2$. By Redei's theorem, this theorem holds for $k=2$. Hence, suppose that $k \geq 3$. Assume w.l.o.g. that $T$ contains the arc $a=(23 \ldots n)$. Let $b$ be the arc of $T$ that has the vertices $1,2, \ldots, n-1$. Consider the $k$-1-hypertournament $T^{\prime}=\left(V^{\prime}, A^{\prime}\right)$ obtained from $T$ by deleting the arc $a$, deleting $n$ from the arcs in $A-\{a, b\}$, and finally deleting 1 from $b$. So, $V^{\prime}=\{1,2, \ldots, n-1\}$, $A^{\prime}=\left\{e^{\prime}: e^{\prime}\right.$ is $e$ without $\left.n, e \in A-\{a, b\}\right\} \cup\left\{b^{\prime}\right\}$, where $b^{\prime}$ is $b$ without the vertex 1. By the induction hypothesis, $T^{\prime}$ has a Hamiltonian path $x_{1} a_{1}^{\prime} x_{2} a_{2}^{\prime} \ldots a_{n-2}^{\prime} x_{n-1}$. This path corresponds to the path $Q=x_{1} a_{1} x_{2} a_{2} \ldots a_{n-2} x_{n-1}$ in $T$. Clearly, $\left\{x_{1}, \ldots, x_{n-1}\right\}=$ $\{1, \ldots, n-1\}$ and $A-\left\{a_{1}, \ldots, a_{n-2}\right\}$ consists of the arc $a$ and another arc $c$.

If $x_{n-1} \neq 1$, then $Q$ an is a Hamiltonian path in $T$. Hence from now on assume that $x_{n-1}=1$. Consider two subcases.

Subcase 1.1: $c \neq b$. If the last vertex of $c$ is $n$, then $Q c n$ is a Hamiltonian path in $T$. Otherwise, $x_{j}$ is the last vertex of $c$ for some $j \leq n-1$. If $j>1$ we replace $a_{j-1}$ by anc in $Q$ in order to obtain a Hamiltonian path in $T$. If $j=1$, then $n c Q$ is a Hamiltonian path in $T$.

Subcase 1.2: $c=b$. If $c \neq\left(x_{n-1} x_{n-2} \ldots x_{1}\right)$ so that $x_{i}$ precedes $x_{i+1}$, for some $i$, $1 \leq i \leq n-2$, in $c$, then $P=Q\left[x_{1}, x_{i}\right] c Q\left[x_{i+1}, x_{n-1}\right]$ is a path in $T$. Since $a_{i} \neq b$, one can construct a Hamiltonian path in $T$ from $P$ as in Subcase 1.1. If $c=\left(x_{n-1} x_{n-2} \ldots x_{1}\right)$, then $Q\left[x_{2}, x_{n-1}\right] c x_{1}$ an is a Hamiltonian path in $T$.

Case 2: $k<n-1$. We proceed by induction on $n \geq 4$. The case $n=4$ (and, hence, $k=2$ ) follows from Redei's theorem. Therefore, suppose that $n \geq 5$. Consider the new $k$-hypertournament $T^{\prime \prime}$ obtained from $T$ by deleting the vertex $n$ along with all arcs in $A$ containing $n$. $T^{\prime \prime}$ has a Hamiltonian path because of either Case 1 if $n=k-2$ or the induction hypothesis, otherwise.

Let $P=x_{1} a_{1} x_{2} a_{2} \ldots a_{n-2} x_{n-1}$ be a Hamiltonian path in $T^{\prime \prime}$. If $T$ has an arc $a \in$ $A_{T}\left(x_{n-1}, n\right)$, then Pan is a Hamiltonian path in $T$. Suppose that $A_{T}\left(x_{n-1}, n\right)=\emptyset$. 
Then either $\cup_{i=1}^{n-1} A_{T}\left(x_{i}, n\right)=\emptyset$, or there is an $i$ so that $T$ has no arc where any $x_{j}$, $j>i$ precedes $n$ and $T$ contains an arc $b$ where $x_{i}$ precedes $n$. In the first case, $n c P$ is a Hamiltonian path in $T$, where $c$ is an arc of $T$ containing both $x_{1}$ and $n$. In the second case, $P\left[x_{1}, x_{i}\right]$ bnd $P\left[x_{i+1}, x_{n-1}\right]$ is a Hamiltonian path in $T$, where $d$ is an arc of $T$ containing both $x_{i+1}$ and $n$ and distinct from $b$.

\section{Hamiltonian cycles}

Clearly, every Hamiltonian hypertournament is strong. In this section, we prove that every strong $k$-hypertournament with $n$ vertices, where $3 \leq k \leq n-2$, is Hamiltonian.

However, for every $k \geq 3$, there exists a strong $k$-hypertournament with $n=k+$ 1 vertices which is not Hamiltonian. Indeed, let the $n-1$-hypertournament $H_{n}$ have vertex set $\left\{x_{1}, \ldots, x_{n}\right\}$ and arc set $\left\{a_{1}, a_{2}, \ldots, a_{n}\right\}$, where $a_{1}=\left(x_{2} x_{3} \ldots x_{n-2} x_{n} x_{n-1}\right), a_{2}=$ $\left(x_{1} x_{3} x_{4} \ldots x_{n}\right), a_{3}=\left(x_{1} x_{2} x_{4} x_{5} \ldots x_{n}\right), a_{4}=\left(x_{2} x_{3} x_{1} x_{5} x_{6} \ldots x_{n}\right)$, and

$$
a_{i}=\left(x_{1} x_{2} \ldots x_{i-4} x_{i-3} x_{i-1} x_{i-2} x_{i+1} x_{i+2} \ldots x_{n}\right) \text { for } 5 \leq i \leq n \text {. }
$$

The hypertournament $H_{n}$ is strong because of the following paths: $x_{i} a_{2} x_{j}$ for all $i<j, 2 \notin\{i, j\}, x_{1} a_{3} x_{2}, x_{2} a_{1} x_{j}$ for all $j>2 ; x_{j} a_{j+1} x_{j-1} a_{j} x_{j-2} \ldots x_{i+1} a_{i+2} x_{i}$ for all $3 \leq i<j \leq n$, where $a_{n+1}=a_{1}, x_{j} a_{j+1} x_{j-1} \ldots x_{4} a_{5} x_{3} a_{4} x_{1} a_{3} x_{2}$ for all $3 \leq j \leq n, x_{2} a_{4} x_{1}$, and $x_{j} a_{j+1} x_{j-1} \ldots x_{3} a_{4} x_{1}$ for all $3 \leq j \leq n$.

However, $H_{n}$ is not Hamiltonian. To prove that, assume that $H_{n}$ has a Hamiltonian cycle $C$. We will try to construct $C$ starting from the vertex $x_{n}$. Since $a_{1}$ is the only arc which has a vertex that succeeds $x_{n}, C$ has the form $x_{n} a_{1} x_{n-1} \ldots$. Since $a_{n}$ is the only arc which has a vertex different from $x_{n}$ that succeeds $x_{n-1}, C=x_{n} a_{1} x_{n-1} a_{n} x_{n-2} \ldots$ Continuing this process, we obtain that $C=x_{n} a_{1} x_{n-1} \ldots x_{4} a_{5} x_{3} \ldots$ The only arc where $x_{3}$ precedes $x_{1}$ or $x_{2}$ is $a_{4}$. Hence, $C=x_{n} a_{1} x_{n-1} \ldots x_{4} a_{5} x_{3} a_{4} x_{1} \ldots$. Now we need to include $x_{2}$, $a_{3}$ and $a_{2}$ into $C$. However, this is impossible because only one of the $\operatorname{arcs} a_{3}, a_{2}$ contains $x_{2}$.

The majority digraph $M(H)$ of a $k$-hypertournament with $n$ vertices $H$ has the same vertex set $V$ as $H$ and, for every pair $x, y$ of distinct vertices in $V$, the $\operatorname{arc} x y$ is in $M(H)$ iff $A_{H}(x, y) \geq A_{H}(y, x)$ (or, by $(1),\left|A_{T}(x, y)\right| \geq \frac{1}{2}\left(\begin{array}{l}n-2 \\ k-2\end{array}\right)$ ). Obviously, $M(H)$ is a semicomplete digraph, i.e. every pair of vertices in $M(H)$ is adjacent.

Let $C_{1}, C_{2}, \ldots, C_{t}$ be the strong components of $M(H)$ such that there is no arc from $V\left(C_{j}\right)$ to $V\left(C_{i}\right)$ if $1 \leq i<j \leq t$ (if $M(H)$ is strong, then $t=1$ ). Define the function cn (component number) such that $c n(x)=r$ if $x \in V\left(C_{r}\right)$. We say that $(P, Q)$ is a 
Hamiltonian pair of paths, if $P$ is a $(x, y)$-path in $H$ and $Q$ is a $(y, x)$-path in $M(H)$ such that $V(P) \cup V(Q)=V(H), V(P) \cap V(Q)=\{x, y\}$ and if $M(H)$ is not strong, then $c n(y)<c n(x)$.

The main result of this section is the following:

Theorem 3.1 Every strong $k$-hypertournament with $n$ vertices, where $3 \leq k \leq n-2$, contains a Hamiltonian cycle.

Theorem 3.1 follows immediately from Lemma 3.3 (the case $k=3$ ), Lemma 3.4 (the case $k \geq 4$ and $n \geq 7$ ) and Lemma 3.6 (the remaining case $k=4$ and $n=6$ ). Proofs of these lemmas are given in the rest of this section and based on the following:

Lemma 3.2 For every strong k-hypertournament with $n$ vertices $H$, there exists a Hamiltonian pair of paths.

Proof: Suppose first that $M(H)$ is not strong. Let $C_{1}\left(C_{t}\right.$, resp.) be the first (terminal, resp.) strong component of $M(H)$. Since $H$ is strong there exists a path $P=$ $x_{1} a_{1} x_{2} a_{2} \ldots a_{m-1} x_{m}$ from $C_{t}$ to $C_{1}$ in $H$. Suppose that $P$ is a shortest such path. Then, $x_{1} \in V\left(C_{t}\right), x_{m} \in V\left(C_{1}\right)$ and $\left\{x_{2}, x_{3}, \ldots, x_{m-1}\right\} \cap\left(V\left(C_{1}\right) \cup V\left(C_{t}\right)\right)=\emptyset$. Since $M^{\prime}=M(H)-\left\{x_{2}, x_{3}, \ldots, x_{m-1}\right\}$ is semicomplete and $x_{m}\left(x_{1}\right)$ is in the first (terminal) strong component of $M^{\prime}$, there exists a Hamiltonian path in $M^{\prime}$ from $x_{m}$ to $x_{1}$. Let $Q=y_{1} y_{2} \ldots y_{l}\left(x_{1}=y_{l}\right.$ and $\left.x_{m}=y_{1}\right)$, be such a path. Clearly $(P, Q)$ is a Hamiltonian pair of paths.

Suppose that $M(H)$ is strong. Then, there is a Hamiltonian cycle $R=x_{1} x_{2} \ldots x_{n} x_{1}$ in $M(H)$. Clearly $P=x_{1} a x_{2}$, where $a \in A_{H}\left(x_{1}, x_{2}\right)$, and $Q=R\left[x_{2}, x_{1}\right]$ form a Hamiltonian pair of paths.

Lemma 3.3 Every strong 3-hypertournament with $n$ vertices, where $n \geq 5$, contains a Hamiltonian cycle.

Proof: Let $H$ be a 3-hypertournament with $n \geq 5$ vertices and let $M$ be the majority digraph of $H$.

By Lemma 3.2, there exists a Hamiltonian pair of paths $\left(P_{i}, Q\right)$, where

$P_{i}=x_{1} a_{1} x_{2} a_{2} \ldots a_{i-1} x_{i}$ is a path in $H$ and $Q=x_{i} x_{i+1} \ldots x_{n} x_{1}$ is a path in $M$. Observe that if $u v$ is an arc of $M$, then $\left|A_{H}(u, v)\right| \geq 2$. Since $\left|A_{H}\left(x_{i}, x_{i+1}\right)\right| \geq 2$, there is an $\operatorname{arc} a_{i} \in A_{H}\left(x_{i}, x_{i+1}\right)-a_{i-1}$. The arc $a_{i} \notin\left\{a_{1}, a_{2}, \ldots, a_{i-1}\right\}$, since if $a_{i}=a_{j}$ then $j<i-1$ and the arc $a_{i}$ includes the vertices $x_{j}, x_{j+1}, x_{i}$ and $x_{i+1}$, which is impossible as $H$ is a 3 -hypertournament. Thus, we can extend $P_{i}$ to $P_{i+1}=P_{i} a_{i} x_{i+1}$. Continuing 
this process we obtain a $\left(x_{1}, x_{n}\right)$-path, $P_{n}=x_{1} a_{1} x_{2} \ldots a_{n-1} x_{n}$, which is Hamiltonian, and $\left|A_{H}\left(x_{n}, x_{1}\right)\right| \geq 2$.

If $A_{H}\left(x_{n}, x_{1}\right)=\left\{a_{n-1}, a_{1}\right\}$, then there is an $\operatorname{arc} b \in A_{H}\left(x_{1}, x_{n}\right)$, and $a_{1}=\left(x_{n} x_{1} x_{2}\right)$ and $a_{n-1}=\left(x_{n-1} x_{n} x_{1}\right)$. We now obtain the Hamiltonian cycle $P_{n}\left[x_{2}, x_{n-1}\right] a_{n-1} x_{1} b x_{n} a_{1} x_{2}$.

If $A_{H}\left(x_{n}, x_{1}\right) \neq\left\{a_{n-1}, a_{1}\right\}$, then there is an arc $b \in A_{H}\left(x_{n}, x_{1}\right)-\left\{a_{n-1}, a_{1}\right\}$. As before we see that $b \notin\left\{a_{1}, a_{2}, \ldots, a_{n-1}\right\}$, and therefore we get the Hamiltonian cycle $P_{n} b x_{1}$.

Lemma 3.4 Every strong $k$-hypertournament with $n$ vertices, where $4 \leq k \leq n-2$ and $n \geq 7$, contains a Hamiltonian cycle.

Proof: It is easy to check that, for $4 \leq k \leq n-2,\left(\begin{array}{c}n-2 \\ k-2\end{array}\right) \geq 2 n-4$ if and only if $n \geq 7$. Let $H$ be a $k$-hypertournament with $n$ vertices, such that $4 \leq k \leq n-2$ and $\left(\begin{array}{l}n-2 \\ k-2\end{array}\right) \geq 2 n-4$, and let $M=M(H)$ be the majority digraph of $H$. Now consider the following two cases.

Case 1: $M$ is not strong. Let $C_{1}\left(C_{t}\right)$ be the first (terminal) strong component of $M$. We first prove that $H$ has a pair of distinct vertices $x, y$ such that

$$
\text { there exists a Hamiltonian }(x, y) \text {-path in } H \text { and }\left|A_{H}(y, x)\right| \geq n-1 \text {. }
$$

By Lemma 3.2, there exists a Hamiltonian pair of paths $(P, Q)$, where

$P=x_{1} a_{1} x_{2} a_{2} \ldots a_{m-1} x_{m}$ is a path in $H, x_{1} \in V\left(C_{t}\right), x_{m} \in V\left(C_{1}\right)$, and $Q=y_{1} y_{2} \ldots y_{l}$ is a path in $M$. Recall that $y_{1}=x_{m}$ and $y_{l}=x_{1}$.

We may assume w.l.o.g that, for some $i>1$, cn $\left(y_{i}\right)<c n\left(y_{i+1}\right)$ (the case $i=1$ can be considered analogously). It follows from the definition of $M$ that

$$
\begin{gathered}
\left|A_{H}\left(y_{j}, y_{j+1}\right)\right| \geq n-2 \text { for } j=1,2, \ldots, l-1, \\
\left|A_{H}\left(y_{p}, y_{q}\right)\right| \geq n-1 \text { for } 1 \leq p \leq i<q \leq l .
\end{gathered}
$$

If $l=2$, then $P$ is a path satisfying (2). Hence we may assume that $l>2$. By (3), we can extend the path $P$ to a path $R=r_{1} b_{1} r_{2} b_{2} \ldots b_{n-2} r_{n-1}$ in $H$ with $r_{1}=$ $y_{i+1}, r_{n-1}=y_{i-1}, V(R)=V(H)-y_{i}$. If there is an arc in $H$ in which $y_{i-1}$ precedes $y_{i}$ and which is not already used in $R$, then we can find a Hamiltonian $\left(y_{i+1}, y_{i}\right)$-path in $H$ and $\left|A_{H}\left(y_{i}, y_{i+1}\right)\right| \geq n-1$, therefore we may assume that $A_{H}\left(y_{i-1}, y_{i}\right)=A(R)$.

Since $A\left(y_{i-1}, y_{i}\right)=A(R)$, we observe that $b_{n-2}$ contains the vertices $r_{n-2}, y_{i-1}, y_{i}$ (in that order), thus, $b_{n-2} \in A_{H}\left(r_{n-2}, y_{i}\right)$. Let $c$ be an arbitrary arc in $A_{H}\left(y_{i}, y_{i-1}\right)$ $\left(A_{H}\left(y_{i}, y_{i-1}\right) \neq \emptyset\right.$ since $\left.\left|A_{H}\left(y_{i}, y_{i-1}\right)\right| \geq n-2\right)$. We now obtain a path 
$R^{\prime}=R\left[r_{1}, r_{n-2}\right] b_{n-2} y_{i} c y_{i-1}$ which satisfies (2) because of (4). Thus the claim (2) is completely proved.

Let $S=s_{1} d_{1} s_{2} d_{2} \ldots d_{n-1} s_{n}$ be a Hamiltonian path in $H$ such that $\left|A_{H}\left(s_{n}, s_{1}\right)\right| \geq n-1$.

If $A_{H}\left(s_{n}, s_{1}\right) \neq A(S)$ then there is an arc $e \in A_{H}\left(s_{n}, s_{1}\right)-A(S)$, since $\left|A_{H}\left(s_{n}, s_{1}\right)\right| \geq$ $n-1$ and $|A(S)|=n-1$. Now $S e s_{1}$ is a Hamiltonian cycle in $H$.

If $A_{H}\left(s_{n}, s_{1}\right)=A(S)$, then $\left|A_{H}\left(s_{n}, s_{1}\right)\right|=n-1$. Let $f$ be an arbitrary arc in $A_{H}\left(s_{1}, s_{n}\right)\left(A_{H}\left(s_{1}, s_{n}\right)\right.$ is not empty since $\left.\left|A_{H}\left(s_{1}, s_{n}\right)\right| \geq n-3 \geq 3\right)$. Since $A_{H}\left(s_{n}, s_{1}\right)=$ $A(S)$, it follows that $d_{n-1} \in A_{H}\left(s_{n-1}, s_{1}\right)$ and $d_{1} \in A_{H}\left(s_{n}, s_{2}\right)$. This implies that $S\left[s_{2}, s_{n-1}\right] d_{n-1} s_{1} f s_{n} d_{1} s_{2}$ is a Hamiltonian cycle of $H$.

Case 2: $\mathrm{M}$ is strong. There is a Hamiltonian cycle, $C=x_{1} x_{2} \ldots x_{n} x_{1}$, in $M$. Since $k \geq 4$, there exist distinct $\operatorname{arcs} a_{1}$ and $a_{2}$, such that $\left\{a_{1}, a_{2}\right\} \subseteq A_{H}\left(x_{1}, x_{2}\right)$. Since $k \geq 4$ and $a_{1}$ and $a_{2}$ cannot include exactly the same vertices, either $a_{1}$ or $a_{2}$ does not contain at least one vertex from the set $\left\{x_{4}, x_{5}, \ldots, x_{n-1}\right\}$. Assume w.l.o.g. that $x_{i} \notin a_{1}$, where $i \in\{4,5, \ldots, n-1\}$. Since $\left|A_{H}\left(x_{j}, x_{j+1}\right)\right| \geq n-2$ for all $j=1,2, \ldots, n-1$ we can find distinct arcs in $H, b_{1}, b_{2}, \ldots, b_{n-3}$, such that the following sequence is a path in $H$ :

$$
P=x_{i+1} b_{1} x_{i+2} b_{2} \ldots x_{n} b_{n-i} x_{1} a_{1} x_{2} b_{n-i+1} x_{3} \ldots b_{n-3} x_{i-1} .
$$

Since $a_{1} \notin A_{H}\left(x_{i-1}, x_{i}\right)$ and $\left|A_{H}\left(x_{i-1}, x_{i}\right)\right| \geq n-2$, there is an $\operatorname{arc} b_{n-2} \in A_{H}\left(x_{i-1}, x_{i}\right)-$ $A(P)$.

If $A_{H}\left(x_{i}, x_{i+1}\right) \neq\left\{b_{1}, b_{2}, \ldots, b_{n-2}\right\}$, then let $b_{n-1} \in A_{H}\left(x_{i}, x_{i+1}\right)-\left\{b_{1}, b_{2}, \ldots, b_{n-2}\right\}$ be arbitrary. Now $P b_{n-2} x_{i} b_{n-1} x_{i+1}$ is a Hamiltonian cycle in $H$.

If $A_{H}\left(x_{i}, x_{i+1}\right)=\left\{b_{1}, b_{2}, \ldots, b_{n-2}\right\}$, then let $c \in A_{H}\left(x_{i+1}, x_{i}\right)$ be arbitrary. Observe that $b_{1} \in A_{H}\left(x_{i}, x_{i+1}\right) \cup A_{H}\left(x_{i+1}, x_{i+2}\right)$, thus, $b_{1} \in A_{H}\left(x_{i}, x_{i+2}\right)$, and $b_{n-2} \in A_{H}\left(x_{i-1}, x_{i}\right) \cup$ $A_{H}\left(x_{i}, x_{i+1}\right)$, thus, $b_{n-2} \in A_{H}\left(x_{i-1}, x_{i+1}\right)$. We now obtain the Hamiltonian cycle

$$
P\left[x_{i+2}, x_{i-1}\right] b_{n-2} x_{i+1} c x_{i} b_{1} x_{i+2},
$$

where we define $x_{n+1}=x_{1}$ (when $\left.i=n-1\right)$.

In the rest of this section we adopt the following: $H$ is a strong 4-hypertournament with 6 vertices and $M=M(H)$ is the majority digraph of $H$.

To prove Lemma 3.6, we need one more lemma.

Lemma 3.5 If $M$ contains a Hamiltonian path $P=x_{1} x_{2} x_{3} x_{4} x_{5} x_{6}$ such that $A_{H}\left(x_{6}, x_{1}\right) \neq$ $\emptyset$, then $H$ is Hamiltonian. 
Proof: To show that $H$ is Hamiltonian, it is sufficient to prove that the family of sets $A_{1}, A_{2}, \ldots, A_{6}$, where $A_{i}=A_{H}\left(x_{i}, x_{i+1}\right)$ for $1 \leq i \leq 5$ and $A_{6}=A_{H}\left(x_{6}, x_{1}\right)$, has a system of distinct representatives (arcs of $H$ ). By P. Hall's matching theorem, such a system exists iff

$$
\left|\cup_{r \in R} A_{r}\right| \geq|R| \text { for all subsets } R \text { of }\{1,2,3,4,5,6\} .
$$

If $|R| \leq 3$, then (5) holds by the definition of $M\left(\left|A_{i}\right| \geq 3\right.$ for all $\left.1 \leq i \leq 5\right)$. If $4 \leq|R| \leq 5$, then $R$ contains two integers $i, j$ such that $1<i+1<j \leq 5$. Obviously, $\left|A_{i} \cap A_{j}\right| \leq 1,\left|A_{i}\right|,\left|A_{j}\right| \geq 3$. Hence, $\left|\cup_{r \in R} A_{r}\right| \geq\left|A_{i} \cup A_{j}\right| \geq 5$.

If $|R|=6$, then

$$
\left|\cup_{r \in R} A_{r}\right| \geq\left|A_{1} \cup A_{3} \cup A_{5}\right| \geq 6 .
$$

Lemma 3.6 Every strong 4-hypertournament with 6 vertices contains a Hamiltonian cycle.

Proof: Assume that $H$ is not Hamiltonian.

Assume that $M$ is strong. Since $M$ is semicomplete, $M$ has a Hamiltonian cycle. Hence, $H$ is Hamiltonian by Lemma 3.5. Therefore, we may and will assume that $M$ is not strong.

Let $(P, Q)$ be a Hamiltonian pair of paths such that $P$ has maximum possible length and let $P=x_{1} a_{1} x_{2} a_{2} \ldots a_{m-1} x_{m}, Q=y_{1} y_{2} \ldots y_{l}\left(l=8-m, x_{1}=y_{l}\right.$ and $\left.x_{m}=y_{1}\right)$. If $l \geq 3$, then assume w.l.o.g. that $c n\left(y_{2}\right)<c n\left(y_{l}\right)$ (otherwise $c n\left(y_{1}\right)<c n\left(y_{l-1}\right)$, so we may reverse all arcs). Since $y_{1} y_{2}$ and $y_{l-1} y_{l}$ are arc in $M$, we have $\left|A_{H}\left(y_{1}, y_{2}\right)\right| \geq 3$ and $\left|A_{H}\left(y_{l-1}, y_{l}\right)\right| \geq 3$. By the maximality of $P$ and the fact that $H$ is not Hamiltonian, we conclude that

$$
A_{H}\left(y_{1}, y_{2}\right) \subseteq A(P), A_{H}\left(y_{l-1}, y_{l}\right) \subseteq A(P) .
$$

Since $m-1=|A(P)| \geq\left|A_{H}\left(y_{1}, y_{2}\right)\right| \geq 3$, we obtain $4 \leq m \leq 6$. Consider the following three cases depending on the value of $m$.

Case $1(m=4)$ : $\operatorname{By}\left|A_{H}\left(y_{1}, y_{2}\right)\right| \geq 3,\left|A_{H}\left(y_{l-1}, y_{l}\right)\right| \geq 3$ and (6), we conclude that $A_{H}\left(y_{1}, y_{2}\right)=A_{H}\left(y_{l-1}, y_{l}\right)=\left\{a_{1}, a_{2}, a_{3}\right\}$. The last formula and $\left|\left\{y_{1}, y_{2}, y_{3}, y_{4}\right\}\right|=4=k$ imply that $a_{1}, a_{2}, a_{3}$ consist of the same vertices, which is impossible. 
Case $2(m=5)$ : By $(6)$ and since $|A(P)|<6$, there exists an arc $d \in\left(A_{H}\left(y_{1}, y_{2}\right) \cup\right.$ $\left.A_{H}\left(y_{2}, y_{1}\right)\right)-A(P)=A_{H}\left(y_{2}, y_{1}\right)-A(P)$. If $a_{4} \in A_{H}\left(y_{1}, y_{2}\right)$ then $\left(x_{1} a_{1} x_{2} a_{2} x_{3} a_{3} x_{4} a_{4} y_{2} d y_{1}, y_{1} y_{3}\right)$ is a Hamiltonian pair of paths (since $c n\left(y_{1}\right)<c n\left(y_{3}\right)$ ). This contradicts the maximality of $m$. Therefore $a_{4} \notin A_{H}\left(y_{1}, y_{2}\right)$ which together with (6) and $\left|A_{H}\left(y_{1}, y_{2}\right)\right| \geq 3$ implies that $A_{H}\left(y_{1}, y_{2}\right)=\left\{a_{1}, a_{2}, a_{3}\right\}$. Now, the last formula, (6) and $\left|A_{H}\left(y_{2}, y_{3}\right)\right| \geq 3$ imply that at least two of $a_{1}, a_{2}, a_{3}$ contain all of $y_{1}, y_{2}, y_{3}$. However, $a_{2}$ and $a_{3}$ contain at least two vertices which are not in $\left\{y_{1}, y_{2}, y_{3}\right\}$, a contradiction (as $k=4$ ).

Case $3(m=6)$ : By the definition of a Hamiltonian pair of paths, we have that $c n\left(x_{6}\right)<c n\left(x_{1}\right)$, which implies that $\left|A_{H}\left(x_{6}, x_{1}\right)\right| \geq 4$. Suppose that $\left\{a_{1}, a_{5}\right\} \subseteq A_{H}\left(x_{6}, x_{1}\right)$. By (6), there is an arc $c_{1} \in A_{H}\left(x_{1}, x_{6}\right)-A(P)$. Thus, $x_{1} c_{1} x_{6} a_{1} x_{2} a_{2} x_{3} a_{3} x_{4} a_{4} x_{5} a_{5} x_{1}$ is a Hamiltonian cycle in $H$. This implies that $\left\{a_{1}, a_{5}\right\} \nsubseteq A_{H}\left(x_{6}, x_{1}\right)$. Assume w.l.o.g. that $A_{H}\left(x_{6}, x_{1}\right)=\left\{a_{1}, a_{2}, a_{3}, a_{4}\right\}$ (otherwise $A_{H}\left(x_{6}, x_{1}\right)=\left\{a_{2}, a_{3}, a_{4}, a_{5}\right\}$ and reverse all arcs). Observe that $a_{1}$ includes the vertices $\left\{x_{1}, x_{2}, x_{6}\right\}, \overline{a_{2}}=\left\{x_{1}, x_{2}, x_{3}, x_{6}\right\}, \overline{a_{3}}=$ $\left\{x_{1}, x_{3}, x_{4}, x_{6}\right\}, \overline{a_{4}}=\left\{x_{1}, x_{4}, x_{5}, x_{6}\right\}$, and $a_{5}$ includes the vertices $\left\{x_{5}, x_{6}\right\}$.

Suppose that $\left|A_{H}\left(x_{5}, x_{1}\right)\right| \geq 3$. Observe that $\left|A_{H}\left(x_{1}, x_{6}\right)\right|=2$ and $A_{H}\left(x_{1}, x_{6}\right) \cap$ $\left\{a_{1}, a_{2}, a_{3}, a_{4}\right\}=\emptyset$. Therefore there exists an arc $c_{1} \in A_{H}\left(x_{5}, x_{1}\right)-\left\{a_{1}, a_{4}\right\}$ and an arc $c_{2} \in A_{H}\left(x_{1}, x_{6}\right)-\left\{a_{1}, a_{2}, a_{3}, a_{4}, c_{1}\right\}$. Note that $c_{1} \notin\left\{a_{2}, a_{3}\right\}$. This implies that $x_{1} c_{2} x_{6} a_{1} x_{2} a_{2} x_{3} a_{3} x_{4} a_{4} x_{5} c_{1} x_{1}$ is a Hamiltonian cycle in $H$, a contradiction.

Therefore, $\left|A_{H}\left(x_{5}, x_{1}\right)\right|<3$. This implies that $x_{1} x_{5}$ is in $M$, thus, $c n\left(x_{5}\right) \geq c n\left(x_{1}\right)$. We note that $A_{H}\left(x_{1}, x_{2}\right) \subseteq\left\{a_{1}, a_{2}, a_{5}\right\}$, since we could otherwise find an $\operatorname{arc} c_{1} \in A_{H}\left(x_{1}, x_{2}\right)-$ $\left\{a_{1}, a_{2}, a_{3}, a_{4}, a_{5}\right\}$, such that $x_{1} c_{1} x_{2} a_{2} x_{3} a_{3} x_{4} a_{4} x_{5} a_{5} x_{6} a_{1} x_{1}$ is a Hamiltonian cycle in $H$. Analogously, we can show that $A_{H}\left(x_{2}, x_{3}\right) \subseteq\left\{a_{1}, a_{2}, a_{5}\right\}, A_{H}\left(x_{3}, x_{4}\right) \subseteq\left\{a_{3}, a_{5}\right\}$ and $A_{H}\left(x_{4}, x_{5}\right) \subseteq\left\{a_{4}, a_{5}\right\}$. This implies that $\operatorname{cn}\left(x_{1}\right) \geq \operatorname{cn}\left(x_{2}\right) \geq \operatorname{cn}\left(x_{3}\right) \geq \operatorname{cn}\left(x_{4}\right) \geq \operatorname{cn}\left(x_{5}\right) \geq$ $c n\left(x_{1}\right)$, which in turn yields $c n\left(x_{1}\right)=c n\left(x_{2}\right)=c n\left(x_{3}\right)=c n\left(x_{4}\right)=c n\left(x_{5}\right)$. Hence, $M$ contains exactly two strong components: the initial one consists of the vertex $x_{6}$ and the second one contains all other vertices. Since the second strong component has a Hamiltonian cycle, $M$ contains a Hamiltonian $\left(x_{6}, x_{1}\right)$-path. Moreover, $\left|A_{H}\left(x_{1}, x_{6}\right)\right|=2$. Therefore, by Lemma 3.5, $H$ is Hamiltonian, a contradiction.

\section{Complexity of the Hamiltonian cycle problem for $k$-hypertournaments}

It is well known (see [8] for an efficient algorithm) that the Hamiltonian cycle problem for 2-hypertournaments, i.e. (ordinary) tournaments is polynomial time solvable. The aim of this section is to show that the problem remains polynomial time solvable for $k=3$, but becomes NP-complete for every fixed $k \geq 4$.

Let $H=(V, A)$ be a $k$-hypertournament, $A=\left\{a_{1}, \ldots, a_{m}\right\}$. Associate with $H$ the following edge-coloured directed multigraph $D(H)$ : the vertex set of $D(H)$ is $V$; for distinct 
vertices $x, y \in V, D(H)$ has the arc $x y$ of colour $i$ iff $a_{i} \in A_{H}(x, y)$. Clearly, $H$ contains a path from a vertex $x$ to another vertex $y$ iff $D(H)$ has a path $P$ from $x$ to $y$ such that no two arcs in $P$ have the same colour.

Proposition 4.1 The Hamiltonian cycle problem for 3-hypertournaments is polynomial time solvable.

Proof: Let $H$ be a 3-hypertournament. By Theorem 3.1, it suffices to prove that one can check the existence of a path, in $H$, from a vertex $x$ to another vertex $y$ in polynomial time. Construct the edge-coloured directed multigraph $D(H)$ as above. We prove that $H$ has a path from $x$ to $y$ iff $D(H)$ has some $(x, y)$-path. Clearly, if $H$ has a path from $x$ to $y$, then $D(H)$ contains such a path. Suppose that $D(H)$ has a path $Q=x_{1} \ldots x_{p}$ from $x=x_{1}$ to $y=x_{p}$. If $Q$ has no arcs of the same colour, then $Q$ corresponds, in the obvious way, to an $(x, y)$-path of $H$. Suppose that $Q$ contains arcs of the same colour. This means that there exist a subscript $i$ and an integer $j$ such that the $\operatorname{arcs} x_{i-1} x_{i}$ and $x_{i} x_{i+1}$ have the same colour $j$ (these two are the only arcs of colour $j$ which can be in $Q$ ). We can replace $Q$ by the path $Q\left[x_{1}, x_{i-1}\right] Q\left[x_{i+1}, x_{p}\right]$. Continuing this process, we obtain a new path, in $D(H)$, from $x$ to $y$ without repetition of colours. The new path corresponds to an $(x, y)$-path in $H$.

Theorem 4.2 Let $k \geq 4$ be an integer. The Hamiltonian cycle problem for $k$-hypertournaments $(k-H C H T)$ is NP-complete.

Proof: It is easy to see that $k$-HCHT is in NP.

To show that our problem is NP-hard, we first transform the well known problem 3SAT ([7], p. 46) to 4 -HCHT. Let $U=\left\{u_{1}, \ldots, u_{k}\right\}$ be a set of variables, let $C=\left\{c_{1}, \ldots, c_{m}\right\}$ be a set of clauses such that every $c_{i}$ has three literals, and let $v_{i l}$ be the $l$ 'th literal in the clause $c_{i}$. We may and will assume that $m \geq 3$. We shall construct a 4-hypertournament $H$ which is Hamiltonian iff $C$ is satisfiable. Since it is more convenient, we shall actually construct $D=D(H)$ instead of $H$.

We first construct a spanning subgraph $D^{\prime}$ of $D$. The edge-coloured directed multigraph $D^{\prime}$ consists of $m+k+1$ parts: the first $m$ parts $X_{i}^{1}, i=1, \ldots, m$, correspond to the clauses of $C$, the next $k$ parts $X_{i}^{2}, i=1, \ldots, k$, correspond to the variables of $U$, and the last part $X^{3}=X_{1}^{3}$ is auxiliary. Every part $X_{i}^{1}\left(X_{i}^{2}\right)$ consists of vertices $x_{i j}=x_{i j}^{1}$ $\left(y_{i j}=x_{i j}^{2}\right.$, resp.). $X_{1}^{3}$ has two vertices $z_{1}=x_{11}^{3}, z_{2}=x_{12}^{3}$. For a pair of distinct vertices $v, w$ in $D^{\prime}$, we say that $v<w$ if either $v \in X_{i}^{l}, w \in X_{q}^{j}$ such that $l \leq j$ and if $l=j$ then $i<q$, or $v=x_{i j}^{l}, w=x_{i q}^{l}$, where $j<q$.

Note that, in the constructions below, different symbols denote different colours.

Each of the first $m$ parts $X_{i}^{1}(i \in\{1,2, \ldots, m\})$ consists of six vertices $x_{i 1}, \ldots, x_{i 6}$ and the following arcs: there are two arcs from $x_{i 1}$ to $x_{i 2}$, the first of colour $a_{i 1}$ and the second 
of colour $b_{i 1}$; there is an arc from $x_{i 2}$ to $x_{i 3}$ and from $x_{i 4}$ to $x_{i 5}$ of colours $d_{i 1}$ and $d_{i 2}$, respectively; there are three arcs from $x_{i 3}$ to $x_{i 4}$ of colours $a_{i 2}, b_{i 1}$ and $b_{i 2}$; and there are two arcs from $x_{i 5}$ to $x_{i 6}$ of colours $a_{i 3}$ and $b_{i 2}$. Every $X_{i}^{1}$ is connected to $X_{i+1}^{1}$ by the arc $x_{i 6} x_{i+1,1}$ of colour $e_{i}$, for $i=1,2, \ldots, m-1$.

For each $i=1,2, \ldots, k$, let $f_{i 1}^{\prime}$ be the number of appearances of the literal $u_{i}$ in the clauses of $C$, and let $f_{i 2}^{\prime}$ be the number of appearances of the literal $\bar{u}_{i}$ in the clauses of $C$. Define $f_{i j}, i=1,2, \ldots, k, j=1,2$, as follows: $f_{i j}=f_{i j}^{\prime}+1$ if $f_{i j}^{\prime}>0$ and $f_{i j}=0$, otherwise.

Each of the $k$ parts $X_{i}^{2}$ consists of $f_{i}=f_{i 1}+f_{i 2}$ vertices $y_{i 1}, \ldots, y_{i f_{i}}$, and the basic $\operatorname{arcs} y_{i j} y_{i, j+1}$ for every $j=1,2, \ldots, f_{i}-1$ and additional arcs $y_{i 1} y_{i f_{i 1}}, y_{i, f_{i 1}+1} y_{i f_{i}}$ (the first additional arc does not exist if $f_{i 1}=0$ and the second additional arc does not exist if $\left.f_{i 2}=0\right)$. For every $j=1, \ldots, f_{i 1}-1$, the basic arc $y_{i j} y_{i, j+1}$ has colour $a_{q t}$, if the $j$ 'th appearance of the variable $u_{i}$ in $C$ is the $t^{\prime}$ th literal in the $q$ 'th clause $c_{q}$. For every $j=f_{i 1}+1, \ldots, f_{i}-1$, the basic arc $y_{i j} y_{i, j+1}$ has colour $a_{q t}$, if the $j$ 'th appearance of the negation of the variable $u_{i}$ in $C$ is the $t^{\prime}$ th literal in the $q$ 'th clause $c_{q}$. If both $f_{i 1}$ and $f_{i 2}$ are positive, then there exists an arc $y_{i f_{i 1}} y_{i, f_{i 1}+1}$ and its colour is $g_{i}$. The colour of the additional arcs $y_{i 1} y_{i f_{i 1}}$ and $y_{i, f_{i 1}+1} y_{i f_{i}}$ (possibly, only one of these two arcs does exist) is $s_{i}(i=1, \ldots, k)$. Every $X_{i}^{2}$ is connected to $X_{i+1}^{2}$ by the $\operatorname{arc} y_{i f_{i}} y_{i+1,1}$ of colour $p_{i}$.

We say that an arc $y_{i 1} y_{i f_{i 1}}$ corresponds to the literal $u_{i}$ and an arc $y_{i, f_{i 1}+1} y_{i f_{i}}$ corresponds to the literal $\bar{u}_{i}$. We also say that an arc of $D^{\prime}$ of colour $a_{i l}$ corresponds to the literal $v_{i l}$.

The part $X^{3}$ consists of two vertices $z_{1}, z_{2}$ and an arc $z_{1} z_{2}$ of colour $c_{3}$. Add two more arcs: $x_{m 6} y_{11}$ of colour $c_{1}$ and $y_{k f_{k}} z_{1}$ of colour $c_{2}$.

We have obtained the edge-coloured directed multigraph $D^{\prime}$. We shall prove that $D^{\prime}$ has a path $P$ from $x_{11}$ to $z_{2}$ such that no colour in $P$ appears twice iff $C$ is satisfiable.

Suppose first that $D^{\prime}$ has a path $P$ from $x_{11}$ to $z_{2}$ such that no colour in $P$ appears twice. Hence, for every $i=1,2, \ldots, m$, there is at least one arc of colour $a_{i, l_{i}}$ which is in $P\left[x_{11}, x_{m 6}\right]$. Hence, the subpath $P\left[y_{11}, y_{k f_{k}}\right]$ contains the arcs of colours $s_{j}$ 's corresponding to the literals $v_{i, l_{i}}, i=1,2, \ldots, m$. It follows that if the negation of $v_{i, l_{i}}$ is also in $C$, then the arcs of colours $a_{j q}$ 's of $D^{\prime}$ corresponding to the negation of $v_{i, l_{i}}$ and belonging to $P$ must be in $P\left[y_{11}, y_{k f_{k}}\right]$ and must not be in $P\left[x_{11}, x_{m 6}\right]$. This fact allows us to assign "true" to every literal $v_{i, l_{i}}, i=1,2, \ldots, m$ such that there is an arc of colour $a_{i l_{i}}$ belonging to $P\left[x_{11}, x_{m 6}\right]$. This assignment is proper and makes $C$ satisfied.

Suppose now that $C$ is satisfiable and consider a truth assignment $\alpha$ for $U$ that satisfies all the clauses in $C$. Let $v_{i, l_{i}}, i=1,2, \ldots, m$ be true under $\alpha$. Then the $\operatorname{arcs} x_{i, 2 l_{i}-1} x_{i, 2 l_{i}}, i=$ $1,2, \ldots, m$ and the arcs of colours $s_{r}$ 's corresponding to $v_{i, l_{i}}, i=1,2, \ldots, m$ can be easily extended to a path $P$ from $x_{11}$ to $z_{2}$ such that no colour in $P$ appears twice.

Now we construct $D$ from $D^{\prime}$. Choose any four vertices $v_{1}, v_{2}, v_{3}, v_{4}$ in $D^{\prime}$ such that $v_{1}<v_{2}<v_{3}<v_{4}$. In $D$, the four vertices together with some arcs between them must form 
a monochromatic transitive 4-tournament such that the colour of this tournament differs from the colours of all other such transitive 4 -tournaments. So, we shall add some arcs to $D^{\prime}$ in order to meet this condition. The symbol $T T\left(v_{i_{1}} v_{i_{2}} v_{i_{3}} v_{i_{4}}\right)$ will denote the transitive 4-tournament with vertex set $\left\{v_{i_{1}}, v_{i_{2}}, v_{i_{3}}, v_{i_{4}}\right\}$ and arc set $\left\{v_{i_{p}} v_{i_{q}}: 1 \leq q<p \leq 4\right\}$.

If $D^{\prime}$ contains $\operatorname{arcs} v_{1} v_{2}$ and $v_{3} v_{4}$ of the same colour $c\left(a_{i j}\right.$ or $b_{i j}$ or $\left.s_{i}\right)$, then let $v_{1}, v_{2}, v_{3}, v_{4}$ form in $D$ the tournament $T T\left(v_{3} v_{4} v_{1} v_{2}\right)$ of colour $c$. Otherwise, if $\left\{v_{3}, v_{4}\right\} \neq$ $\left\{z_{1}, z_{2}\right\}$, then $v_{1}, v_{2}, v_{3}, v_{4}$ form the tournament $T T\left(v_{4} v_{3} v_{2} v_{1}\right)$ of a new colour. If $\left\{v_{3}, v_{4}\right\}=$ $\left\{z_{1}, z_{2}\right\}$ and $D^{\prime}$ has an arc from $v_{1}$ to $v_{2}$ of colour $c$ that appears in $D^{\prime}$ only once, then let $v_{1}, v_{2}, v_{3}, v_{4}$ form the tournament $T T\left(z_{2} z_{1} v_{1} v_{2}\right)$ of colour $c$. If $\left\{v_{3}, v_{4}\right\}=\left\{z_{1}, z_{2}\right\}$ and either $D^{\prime}$ has an arc from $v_{1}$ to $v_{2}$ of colour that appears in $D^{\prime}$ twice or $D^{\prime}$ has no arc from $v_{1}$ to $v_{2}$, but $\left\{v_{1}, v_{2}\right\} \neq\left\{x_{11} x_{21}\right\}$ or $\left\{x_{11} y_{k, f_{k}}\right\}$, then $v_{1}, v_{2}, v_{3}, v_{4}$ form the tournament $T T\left(v_{4} v_{3} v_{2} v_{1}\right)$ of a new colour. If $\left(v_{1}, v_{2}, v_{3}, v_{4}\right)=\left(x_{11}, y_{k f_{k}}, z_{1}, z_{2}\right)\left(\left(v_{1}, v_{2}, v_{3}, v_{4}\right)=\left(x_{11}, x_{21}, z_{1}, z_{2}\right)\right.$, resp.), then $v_{1}, v_{2}, v_{3}, v_{4}$ form the tournament $T T\left(z_{2} y_{k f_{k}} z_{1} x_{11}\right)\left(T T\left(z_{1} z_{2} x_{21} x_{11}\right)\right.$, resp.) of colour $c_{2}\left(c_{3}\right.$, resp.).

Observe that $D$ is an edge-coloured directed multigraph of some 4-hypertournament $H$ and all $\operatorname{arcs} v w$ of $D$ such that $v<w$ are the arcs of $D^{\prime}$. Thus, by the construction of $D, D$ has a path $P$ from $x_{11}$ to $z_{2}$ which contains no arcs of the same colour iff $D^{\prime}$ has such a path. Moreover, if $D$ has such a path $P$, the path $P$ can be extended to a Hamiltonian cycle $W$ in $D$ which contains no arcs of the same colour. Indeed, only some vertices $y_{r}^{\prime}=y_{j_{r}, q_{r}} \in \cup_{i=1}^{k} X_{i}^{2}(r=1,2, \ldots, p)$ are not in $P$. If $p=0$, then we use the arc $z_{2} x_{11}$ of the tournament $T T\left(z_{2} x_{31} x_{21} x_{11}\right)$ to construct $W$. If

$p>0$, then we use the arcs $z_{2} y_{r}^{\prime}, y_{r}^{\prime} y_{r-1}^{\prime}, \ldots, y_{2}^{\prime} y_{1}^{\prime}, y_{1}^{\prime} x_{11}$ of the tournaments $T T\left(z_{2} y_{r}^{\prime} x_{21} x_{11}\right)$, $T T\left(y_{r}^{\prime} y_{r-1}^{\prime} x_{21} x_{11}\right), \ldots, T T\left(y_{2}^{\prime} y_{1}^{\prime} x_{21} x_{11}\right), T T\left(y_{1}^{\prime} x_{31} x_{21} x_{11}\right)$ to construct $W$.

Therefore, the 4-hypertournament $H$ corresponding to $D$ is Hamiltonian iff $C$ is satisfiable. This completes the proof for 4-hypertournaments. One can easily modify the construction of $D$ such that $D$ will correspond to a $q$-hypertournament, $q \geq 5$, using $q-2$ vertices, instead of two, in the last part $X^{3}$ of $D^{\prime}$.

\section{$5 \quad$ Remarks and open problems}

When all results of this paper except Lemma 3.6 were already proved, Susan Marshall informed us (personal communication) that she independently obtained Theorem 2.1 (unpublished).

We have obtained a characterization of Hamiltonian $k$-hypertournaments with $n \geq k+2$ vertices. Yet, we were unable to characterize Hamiltonian $n-1$-hypertournaments with $n$ vertices. Note that a non-difficult modification of the construction in the proof of Theorem 4.2 shows that the Hamiltonian cycle problem for $n$-1-hypertournaments with $n$ vertices is NP-complete. 
It would also be interesting to characterize pancyclic and vertex pancyclic hypertournaments (extensions of well-known theorems by Moser and Moon, respectively, [11]).

\section{Acknowledgments}

The research of GG was supported by the Danish Research Council under Grant No. 11-534-1. This support is gratefully acknowledged.

We would like to thank the referees for very careful reading of the paper and many useful suggestions, which have considerably improved the presentation of the paper. One of the suggestion gave us a possibility to significantly shorten the proof of Lemma 3.6.

\section{References}

[1] R. Assous, Enchainbilite et seuil de monomorphie des tournois n-aires, Discrete Math., 62 (1986) 119-125.

[2] E. Barbut and A. Bialostocki, A generalisation of rotational tournaments, Discrete Math., 76 (1989) 81-87.

[3] E. Barbut and A. Bialostocki, On regular $r$-tournaments, Combinatorica, 34 (1992) 97-106.

[4] C. Berge, Graphs and Hypergraphs. North-Holland, Amsterdam, 1973.

[5] A. Bialostocki, An application of the Ramsey theorem to ordered $r$-tournaments, Discrete Math., 61 (1986) 325-328.

[6] P. Frankl, What must be contained in every oriented $k$-uniform hypergraph, Discrete Math., 62 (1986) 311-313.

[7] M.R. Garey and D.S. Johnson, Computers and Intractability: a Guide to the Theory of NP-Completeness, Freeman, San Fransisco, 1979.

[8] Y. Manoussakis, A linear time algorithm for finding Hamiltonian cycle in tournaments. Discrete Appl. Math. 36 (1992) 199-202.

[9] S. Marshall, On the existence of $k$-tournaments with given automorphism group, Discrete Math. 152 (1996) 259-268.

[10] S. Marshall, Properties of $k$-tournaments, PhD thesis, Simon Fraser University, 1994.

[11] J.W. Moon, Topics on Tournaments. Holt, Rinehart and Winston, N.Y., 1968. 
[12] K.B. Reid, Tournaments: scores, kings, generalizations, and special topics, in Surveys in Graph Theory, G. Chartrand and M. Jacobson (eds), Congr. Numer. 115 (1996), in press. 